

\title{
Un spectromètre de masse équipé d'une source d'ions à excitation électrique de haute fréquence et d'un dispositif électronique digital de traitement d'information: application au dosage isotopique

\author{
C.T. Huynh, J. Garcia, L. Espagno
}

\section{To cite this version:}

C.T. Huynh, J. Garcia, L. Espagno. Un spectromètre de masse équipé d'une source d'ions à excitation électrique de haute fréquence et d'un dispositif électronique digital de traitement d'information: application au dosage isotopique. Revue de Physique Appliquée, 1968, 3 (1), pp.33-45. 10.1051/rphysap:019680030103300 . jpa-00242818

\section{HAL Id: jpa-00242818 https://hal.science/jpa-00242818}

Submitted on 1 Jan 1968

HAL is a multi-disciplinary open access archive for the deposit and dissemination of scientific research documents, whether they are published or not. The documents may come from teaching and research institutions in France or abroad, or from public or private research centers.
L'archive ouverte pluridisciplinaire HAL, est destinée au dépôt et à la diffusion de documents scientifiques de niveau recherche, publiés ou non, émanant des établissements d'enseignement et de recherche français ou étrangers, des laboratoires publics ou privés. 


\title{
UN SPEGTROMÈTRE DE MASSE ÉQUIPÉ D'UNE SOURGE D'IONS A EXGITATION ÉLEGTRIQUE DE HAUTE FRÉQUENGE ET D'UN DISPOSITIF ÉLEGTRONIQUE DIGITAL DE TRAITEMENT D'INFORMATION : APPLIGATION AU DOSAGE ISOTOPIQUE
}

\author{
Par C. T. HUYNH, J. GARCIA et L. ESPAGNO, \\ Laboratoires de Chimie-Physique, Centre de Recherches S.N.P.A., 64-Pau. \\ (Reçu le 19 octobre 1967.)
}

\begin{abstract}
Résumé. - On décrit un spectromètre de masse, équipé d'une source d'ions à excitation électrique de haute fréquence et d'un dispositif électronique digital de traitement d'information. Ce spectromètre de masse ayant un tube séparateur de $10 \mathrm{~cm}$ de rayon et muni de tentes d'entrée et de sortie de $0,5 \times 8 \mathrm{~mm}$, délivre au collecteur d'ions un courant de l'ordre du microampère. Son pouvoir séparateur moyen est, avec ces fentes, égal à 80 .

On étudie les variations du courant ionique collecté pour différents gaz en fonction de deux paramètres principaux : la tension d'accélération des ions et la pression partielle du gaz dans la source. Le courant collecté augmente avec la tension d'accélération, alors que la dispersion en énergie des ions diminue.

On montre également que la variation du courant collecté n'est pas une fonction linéaire de la pression d'introduction du gaz. L'application à des problèmes d'analyse est toutefois possible si on dilue les gaz échantillons dans un gaz inerte (de l'hélium par exemple).

Enfin, on rapporte les résultats obtenus avec cet appareillage sur le dosage en continu des isotopes partiellement séparés par chromatographie en phase gazeuse.

Abstract. - We describe in the present article a mass spectrometer equipped with a high frequency ion source and with an electronic digital device for information processing. This mass spectrometer has a $10 \mathrm{~cm}$ radius separator tube and input and output slits of $0.5 \times 8 \mathrm{~mm}$, and delivers to the ion collector a current of the order of a microampere. Its resolving power is, with these slits, equal to 80 .

The variations of the ion current collected have been studied for different gases with respect to the principal parameters : ion acceleration field and partial gas pressure in the source. The collected current increases with the acceleration field, while the dispersion in ion energy decreases.

Furthermore, it is shown that the variation of collected current is not a linear function of the gas introduction pressure. Application to analysis problems is however possible if the gas samples are diluted in an inert gas (helium for example).

Finally, we report results of the application of this apparatus to the continuous analysis of isotopes partially separated by gas chromatography.
\end{abstract}

Introduction. - Les spectromètres de masse usuels sont équipés de sources d'ions à bombardement électronique dont le modèle le plus courant est dû à Nier : un faisceau d'électrons émis par un filament porté à l'incandescence et possédant une énergie de quelques dizaines d'électron-volts ionise la substance à étudier. Les ions ainsi formés sont accélérés et séparés dans un champ magnétique puis collectés. Le courant ionique total fourni par une telle source d'ions est de l'ordre de $10^{-6}$ ampère à $10^{-8}$ ampère et celui collecté de $10^{-10}$ à $10^{-12}$ ampère. On s'est proposé de construire un spectromètre de masse équipé d'une source à fort courant ionique à ionisation par décharge électrique de haute fréquence décrite par D. Blanc et Degeilh [1].

L'emploi de telles sources d'ions en spectrométrie de masse n'a pas été, à notre connaissance, étudié systématiquement. Les auteurs ayant abordé ce sujet ont, pour la plupart, analysé les réponses de la source données par l'hydrogène et étudié les variations des différents pics correspondant aux rapports $m / e$ égaux à 1,2 et 3 [2], [3]. A noter aussi les essais de Abbe et Adloff [4], [5] qui ont remplacé sur un spectromètre de masse Atlas Werke $\mathrm{CH} 4$ la source de Nier par une source d'ions à haute fréquence. Ils ont signalé 
l'importance des courants ioniques collectés, le maintien d'une bonne résolution avec, en contrepartie, les inconvénients suivants : difficultés d'identification des ions et des mécanismes des réactions, impossibilité d'enregistrer le «bruit de fond » de l'appareil et de mesurer les potentiels d'apparition des ions.

En outre, un dispositif électronique digital de traitement d'information comportant un nombre variable de mémoires et de totalisateurs binaires a été mis au point pour permettre ainsi d'emmagasiner les informations données par le spectromètre de masse sous forme numérique et effectuer éventuellement différentes opérations. Pour des analyses de plusieurs constituants, les mesures isotopiques par exemple, l'appareil construit dispose d'un seul collecteur sur lequel défilent par balayage magnétique les groupes d'ions de différentes masses. Ce collecteur unique est suivi d'un convertisseur analogique-digital et d'un nombre variable de mémoires et de totalisateurs binaires suivant le nombre de produits à doser.

Une application particulière de ce dispositif électronique est faite dans le cas du dosage en continu d'isotopes partiellement séparés par chromatographie en phase gazeuse. Les résultats obtenus sur les isotopes ${ }^{12} \mathrm{C}$ et ${ }^{13} \mathrm{C} \mathrm{du}$ carbone et ${ }^{20} \mathrm{Ne}$ et ${ }^{22} \mathrm{Ne}$ du néon sont en bon accord avec ceux obtenus par la méthode de la double collection.

\section{GHAPITRE I}

\section{DESCRIPTION DU SPECTROMETTRE DE MASSE}

Nous décrivons successivement la source d'ions, les dispositifs d'extraction, de focalisation, de séparation et de collection des ions ainsi que les systèmes de pompage et d'introduction des gaz.

1. Source d'ions. - La source d'ions comporte essentiellement une chambre d'ionisation et un générateur électrique de haute fréquence.

La chambre d'ionisation, de forme cylindrique, est réalisée en pyrex, d'après le modèle proposé par D. Blanc et Degeilh [1] ( fig. 1). Ses dimensions sont les suivantes : longueur, $110 \mathrm{~mm}$; diamètre intérieur, $30 \mathrm{~mm}$; diamètre extérieur, $40 \mathrm{~mm}$; diamètre du canal d'extraction, $7 \mathrm{~mm}$. Les gaz à étudier traversent cette chambre d'ionisation à basse pression (de l'ordre de $10^{-2}$ à $10^{-3}$ torr) où règne un champ électromagnétique de haute fréquence. Sous l'action de ce dernier, ils s'ionisent et forment un plasma. Les ions positifs formés dans ce plasma sont extraits par un dispositif qui sera décrit plus loin.

Le champ électromagnétique de haute fréquence nécessaire à l'ionisation est fourni par un oscillateur qui, par couplage inductif, ionise le gaz se trouvant dans la chambre. Le générateur haute fréquence est un auto-oscillateur classique constitué par une double tétrode QQE 06/40. La bobine oscillatrice du géné-



Fig. 1. - Schéma d'ensemble de la source d'ions (chambre d'ionisation, système d'extraction et de focalisation).

rateur sert en même temps de bobine excitatrice de la source, ce qui améliore le couplage et réduit l'encombrement. Le générateur $\mathrm{HF}$ est réglé pour travailler avec une fréquence de l'ordre de $80 \mathrm{MHz}$ et peut fournir une puissance électrique de haute fréquence de $80 \mathrm{~W}$, valeur déduite à partir des caractéristiques de la lampe oscillatrice données par le constructeur. L'ensemble du montage de l'oscillateur et du ventilateur de refroidissement de la double tétrode et de la source est blindé pour éviter tout rayonnement $\mathrm{HF}$.

2. Extraction et accélération des ions. - Le fonctionnement et la qualité de la source HF sont étroitement liés à la forme et aux dimensions du système d'extraction des ions.

Nous avons utilisé le système d'extraction proposé par D. Blanc et Degeilh représenté sur la figure 1. L'électrode d'extraction $\mathbf{E}_{2}$ faite en aluminium pour éviter la pulvérisation cathodique est vissée sur le support de source $\mathrm{S}$. Elle a un diamètre de $3 \mathrm{~mm}$ et une longueur de $6 \mathrm{~mm}$. L'anode $\mathrm{E}_{1}$ est une tige de 
tungstène de $1,5 \mathrm{~mm}$ de diamètre. L'anode est portée à un potentiel positif haute tension compris entre 1000 et $3000 \mathrm{~V}$, l'électrode $E_{2}$ d'extraction restant à un potentiel de l'ordre de $2000 \mathrm{~V}$.

3. Focalisation des ions. - Le faisceau d'ions extraits à la sortie de la chambre d'ionisation est dans la plupart des cas trop divergent pour être envoyé directement dans le tube analyseur. Nous avons donc cherché à concentrer le faisceau et à réduire son ouverture par un système de focalisation (fig. 1) [6].

L'électrode $\mathrm{E}_{3}$ est au potentiel de la masse. Les électrodes $\mathrm{E}_{4}$ et $\mathrm{E}_{5}$ sont « autopolarisées 》 par l'intermédiaire des résistances $\mathrm{R}_{4}$ et $\mathrm{R}_{5}$. Enfin, l'électrode $\mathrm{E}_{6}$ est portée à un potentiel négatif variable de 300 à $3000 \mathrm{~V}$.

4. Système analyseur, collection des ions. - L'analyse en masse du faisceau d'ions extraits et focalisés s'effectue de façon classique à l'aide d'un système dispersif à induction magnétique. L'analyseur magnétique à champ variable est construit par la compagnie de Télégraphie Sans Fil (G.S.F.). L'électro-aimant est formé de deux enroulements comportant environ 30000 spires traversées par un courant réglable entre 10 et $180 \mathrm{~mA}$. L'induction maximale atteinte dans l'entrefer de $10 \mathrm{~mm}$ est de 0,4 weber $/ \mathrm{m}^{2}$. L'alimentation stabilisée de l'électro-aimant peut être réglée manuellement à une valeur fixe. Un balayage mécanique automatique croissant ou décroissant et un balayage cyclique dont la période et l'étendue sont réglables peuvent également être utilisés.

Le tube analyseur a un rayon de courbure de $10 \mathrm{~cm}$ et un secteur de $90^{\circ}$. L'une de ses extrémités est prolongée par un collecteur d'ions en forme de cage de Faraday portée à un potentiel très voisin de la masse. Une électrode additionnelle qui fait également office de fente d'entrée $(0,5 \times 8 \mathrm{~mm})$ du collecteur est au potentiel négatif de $50 \mathrm{~V}$ pour repousser les électrons secondaires émis par la cage sous l'action du bombardement ionique.

Les courants de l'ordre du microampère $\left(10^{-6} \mathrm{~A}\right)$ sont suffisamment intenses pour attaquer directement un enregistreur galvanométrique SEFRAM dont la sensibilité maximale est de $10^{-9}$ ampère pour $1 \mathrm{~mm}$. On les amplifie avec un amplificateur à courant continu classique.

5. Circulation des gaz. - a) Groupe De pompage. - Le vide dans le tube séparation et dans l'enceinte où est logée l'optique ionique doit être de l'ordre de $10^{-6}$ torr. Ce vide est assuré par un groupe de pompage constitué d'une pompe primaire à palette et d'une pompe secondaire à diffusion de vapeur de mercure.

Il est très important d'avoir une grande vitesse de pompage afin d'obtenir un gradient de pression aussi élevé que possible entre la chambre d'ionisation $\left(10^{-2}\right.$ à $10^{-3}$ torr $)$ et la sortie du canal d'extraction $\left(10^{-5}\right.$ à $10^{-6}$ torr $)$. Nous avons utilisé une pompe secondaire dont la vitesse de pompage est égale à $300 \mathrm{l} / \mathrm{s}$.

b) Système D'introduction des Gaz. - Le système d'introduction "statique " des gaz est classique. Le gaz à analyser est détendu dans un ballon intermédiaire; une fuite réglable permet ensuite de l'introduire dans la source. Le système est à double voie et la pression peut être mesurée dans chacun des ballons de détente. La consommation en gaz étant relativement importante, de l'ordre de 5 à $10 \mathrm{~cm}^{3} / \mathrm{h} 5$ (TPN), afin de travailler sans modification sensible de la pression dans la source d'ions, nous avons pris comme pression de détente 200 torrs et comme volume de chaque ballon $1000 \mathrm{~cm}^{3}$.

Un dispositif d'introduction " dynamique » original a été construit [7] pour permettre la liaison chromatographe-spectromètre de masse. Le chromatographe travaille au voisinage de la pression atmosphérique avec un débit gazeux de l'ordre du centimètre cube par seconde. La source du spectromètre de masse fonctionne à une pression de l'ordre de $10^{-3}$ torr et le débit de gaz est de l'ordre de $10^{3} \mathrm{~cm}^{3} / \mathrm{s}$ à cette pression, c'est-à-dire $10^{-3} \mathrm{~cm}^{3} / \mathrm{s}$ à la pression atmosphérique. Le dispositif qui relie le chromatographe au spectromètre de masse doit réaliser une détente dans le rapport $10^{6}$ et ne prélever en fait qu'une fraction de l'ordre de $10^{-3}$ de flux gazeux sortant de la colonne chromatographique. Ce dispositif d'introduction dynamique comporte deux étages. Un premier capillaire métallique $(L=1 \mathrm{~m}, \varnothing=0,2 \mathrm{~mm})$ est branché sur le chromatographe en amont du détecteur à fils chauds. Il conduit une fraction des effluents à une chambre de détente intermédiaire où débouche un capillaire en verre $(L=10 \mathrm{~mm}, \varnothing=0,04 \mathrm{~mm})$ directement raccordé à la source. Un système de pompage relié par une fuite réglable à la chambre de détente intermédiaire complète le dispositif. La discrimination isotopique liée au régime d'écoulement dans le capillaire de verre est reproductible et d'ailleurs négligeable devant les enrichissements isotopiques que donne la colonne de chromatographie.

\section{GHAPITRE II}

\section{PERFORMANCES DU SPECTROMETTRE DE MASSE REALISE}

La pression de fonctionnement normal est atteinte après trois heures de pompage en partant de la pression atmosphérique. Ge temps est aussi le temps nécessaire pour la stabilisation des organes électroniques de l'appareil. On règle la pression dans la chambre de détente intermédiaire de façon à obtenir dans la source d'ions une pression de l'ordre de quelque $10^{-3}$ torr. Le débit de la pompe à diffusion est suffisant pour maintenir dans l'enceinte à vide et dans le tube analyseur une pression inférieure à $10^{-5}$ torr. La 
décharge HF se stabilise au bout d'un quart d'heure de fonctionnement et elle peut être arrêtée et réamorcée à tout moment. On peut alors faire varier tous les paramètres et obtenir des résultats reproductibles. Le but des réglages est d'obtenir un courant ionique collecté intense, tout en maintenant un pouvoir séparateur élevé.

1. Courant extrait et courant collecté. - A l'aide d'une cible placée directement à la sortie de la source, nous avons mesuré le courant ionique extrait en fonction de la tension d'extraction pour une pression d'air de l'ordre $10^{-2}$ torr dans la source. L'oscillateur haute fréquence fonctionne à $78 \mathrm{MHz}$ avec une tension plaque de $600 \mathrm{~V}$ et un courant plaque de $80 \mathrm{~mA}$.

La figure 2 représente le courant obtenu en fonction de la tension d'extraction. On constate que le courant

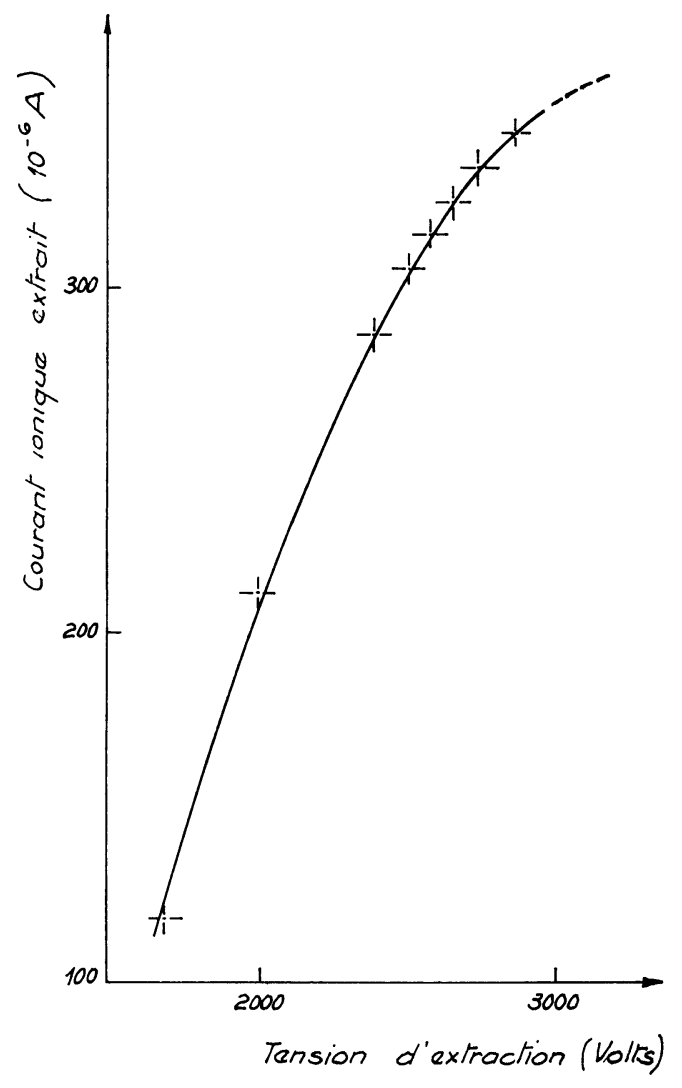

FIG. 2. - Variation du courant ionique collecté en fonction de la tension d'extraction.

ionique est très faible en dessous de $700 \mathrm{~V}$, croît rapidement entre 1000 et $2000 \mathrm{~V}$ et semble, au-dessus de $3000 \mathrm{~V}$, suivre la loi : $I=k\left(V_{\text {ext }}\right)^{b}$ avec $b=0,5$. Ge résultat est en bon accord avec celui obtenu par Degeilh [8] qui déduit de ses mesures expérimentales $b$ voisin de 0,66 dans un domaine de tension d'extraction de 2000 à 10000 V. D'autres gaz (He, Ne, A, $\mathrm{N}_{2}$, etc.) ont conduit à des courbes semblables. Nous avons utilisé par la suite le montage complet, c'est-àdire : système de focalisation et système analyseur. L'ensemble est réglé de manière à obtenir l'intensité maximale du courant collecté pour une pression d'introduction donnée. La tension de focalisation optimale appliquée à l'électrode $\mathrm{E}_{6}$ ( fig. 1) croît avec la tension d'extraction. Le courant recueilli sur le collecteur du spectromètre est de l'ordre de $1 \%$ du courant extrait de la source à $3000 \mathrm{~V}$. Cette proportion décroît rapidement quand la tension d'extraction s'abaisse $(0,3 \%$ à $2000 \mathrm{~V}$ ) [9]. Les conditions de fonctionnement utilisées par la suite sont : $V_{\text {ext }}=3000 \mathrm{~V}$ et $V_{\text {foc }}=-2500 \mathrm{~V}$.

2. Pression dans la source. - Le courant ionique collecté pour différents gaz purs est mesuré en fonction de la pression dans le ballon de détente intermédiaire. On admet que cette dernière est directement proportionnelle à la pression dans la source d'ions, la géométrie : ballon de détente intermédiaire, source d'ions et groupe de pompage, étant donnée.

L'hélium donne un seul pic correspondant à la masse 4, le néon deux principaux dus à ses isotopes ${ }^{20} \mathrm{Ne}$ et ${ }^{22} \mathrm{Ne}$. La figure 3 montre, en échelle doublement logarithmique, les résultats obtenus pour ces deux gaz. On constate que l'intensité du courant

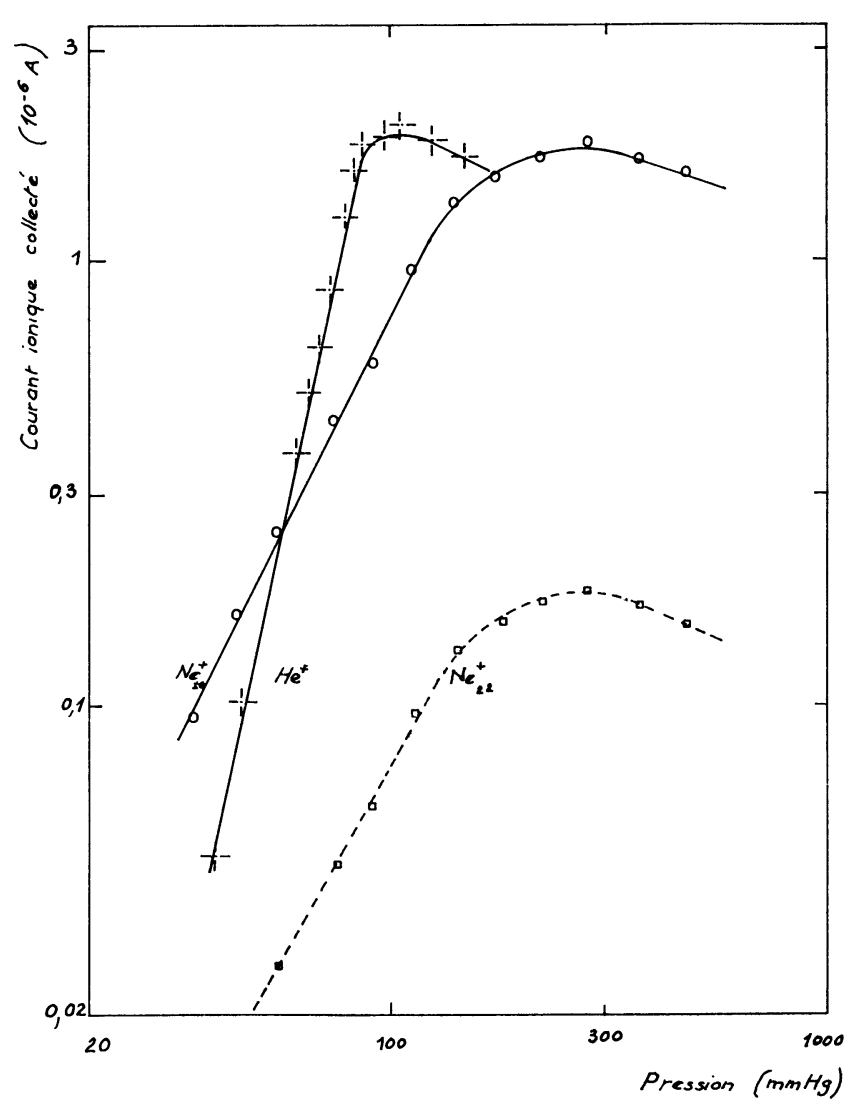

FIG. 3. - Variation du courant ionique collecté en fonction de la pression de néon et d'hélium dans la chambre de détente intermédiaire. 
collecté augmente fortement avec la pression, passe par un maximum, puis décroît, et que le rapport isotopique du néon (22 et 20 ) reste constant.

Il importe de noter que le courant obtenu pour un gaz pur n'est pas proportionnel à la pression, résultats concordant avec ceux de la littérature. De ce fait, les dosages ou analyses quantitatives deviennent pratiquement impossibles. Néanmoins, on peut remédier à cet inconvénient en diluant les gaz étudiés dans un gaz inerte, de l'hélium par exemple. On a vérifié par ailleurs que l'on obtient une réponse linéaire de la source pour différents gaz dilués dans de l'hélium à un taux de l'ordre de $15 \%$ [9].

3. Pouvoir séparateur. - On choisit comme définition du pouvoir séparateur $P$ du spectromètre pour les masses $M$ et $M^{\prime}$ séparées par la distance $L$ :

$$
P=\frac{L}{a} \cdot \frac{M}{M-M^{\prime}}
$$

$a$ étant la largeur du pic à $1 \%$ de sa hauteur.

La figure 4 reproduit la variation du pouvoir séparateur en fonction de la tension d'extraction pour le cas du néon. Il passe d'une valeur voisine de 25 pour $1000 \mathrm{~V}$ de tension d'extraction à 80 pour $3000 \mathrm{~V}$, tension de fonctionnement normal.

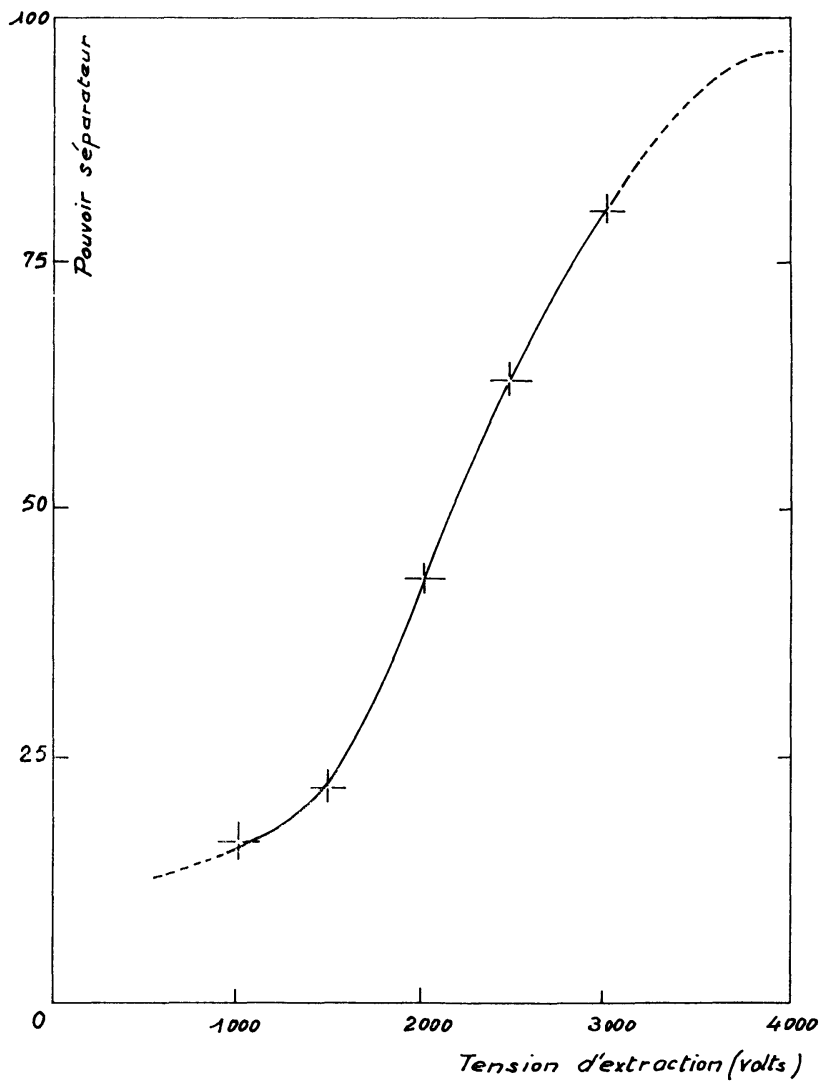

FIG. 4. - Variation du pouvoir séparateur en fonction de la tension d'extraction.
On peut exprimer ce résultat en fonction de la dispersion énergétique $\Delta V / V$ des ions émis par la source, à partir du modèle simplifié de Barnard [10] qui fait intervenir les largeurs des fentes objet et image du secteur magnétique $f_{1}$ et $f_{2}$ et le rayon de courbure $r$ des trajectoires ioniques :

$$
P=\frac{r}{f_{1}+f_{2}+r \cdot \Delta V / V} .
$$

La formule précédente permet de déduire les valeurs de la dispersion énergétique des ions $\Delta V / V$ en fonction des tensions d'extraction. Les résultats ainsi obtenus sont portés sur la figure 5 . On constate que la disper-

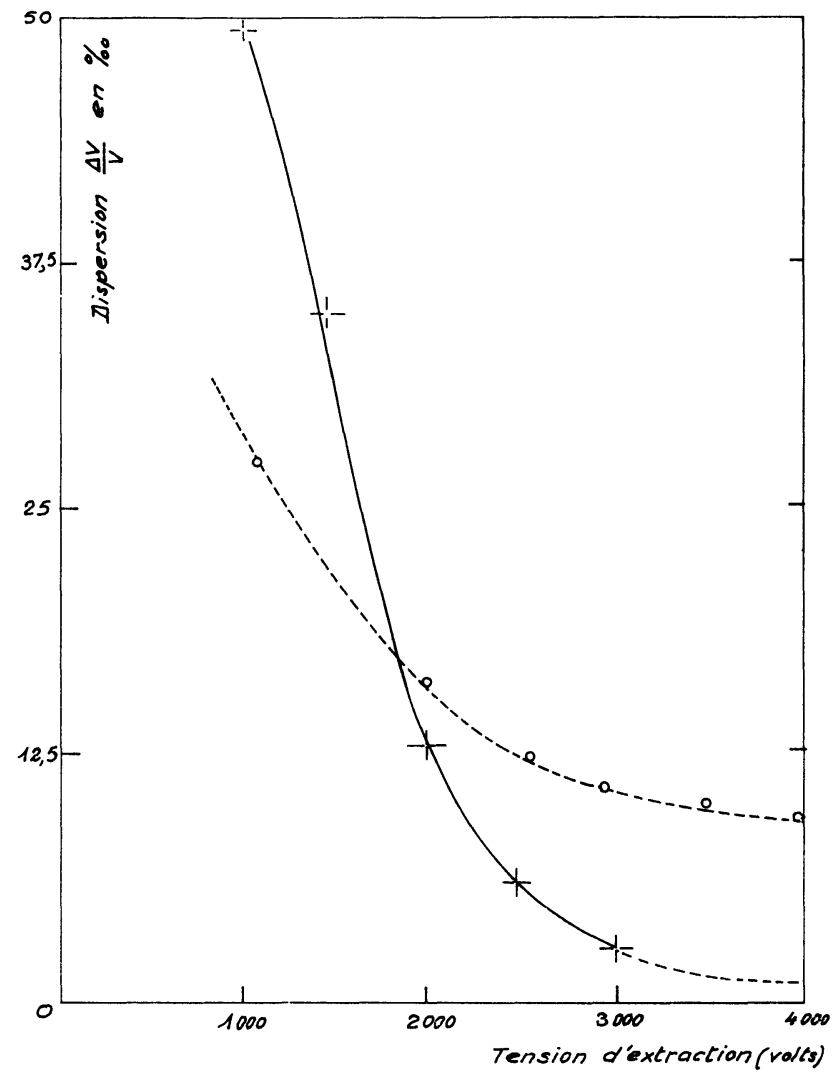

FIG. 5. - Variation de la dispersion énergétique des ions en fonction de la tension d'extraction : construit :

Courbe établie avec le spectromètre de masse

- - - Courbe établie avec le sélecteur de vitesse de Bordenave-Montesquieu.

sion énergétique diminue de $50 \%$ pour une tension d'extraction de $1000 \mathrm{~V}$ à $2,5 \%$ pour une tension d'extraction de $3000 \mathrm{~V}$.

Ces résultats sont à comparer à ceux obtenus par Bordenave-Montesquieu [11] sur la dispersion énergétique des ions donnés par ce type de source. On a reproduit sur la figure 5 les variations de la dispersion $\Delta V / V$ en fonction de la tension d'extraction obte- 
nue par cet auteur à l'aide d'un secteur électrostatique. Cette courbe est semblable à celle obtenue à partir des valeurs du pouvoir séparateur du spectromètre de masse. L'écart entre les deux courbes est sans doute dû au fait que nos mesures ont été effectuées sur le néon avec un système de focalisation des ions et celles de Bordenave-Montesquieu sur l'hélium et sans système de focalisation.

\section{CHAPITRE III}

\section{DISPOSITIF ELECTRONIQUE DIGITAL DE TRAITEMENT D'INFORMATION}

Le spectromètre de masse construit est équipé d'un dispositif électronique digital de traitement des pics de masse. Ce dernier comporte essentiellement un convertisseur analogique-digital, un nombre variable de mémoires et de totalisateurs binaires suivant le nombre de produits à doser et une logique de commande.

Le schéma de principe du dispositif électronique est représenté sur la figure 6 . Un commutateur automatique de sensibilités normalise les pics spectrométriques. Un convertisseur analogique-digital traduit chaque pic en digits binaires qui sont emmagasinés dans des mémoires ou des totalisateurs distincts. Une logique de commande agit sur le commutateur de sensibilités, suit les opérations de codage, distribue les ordres de

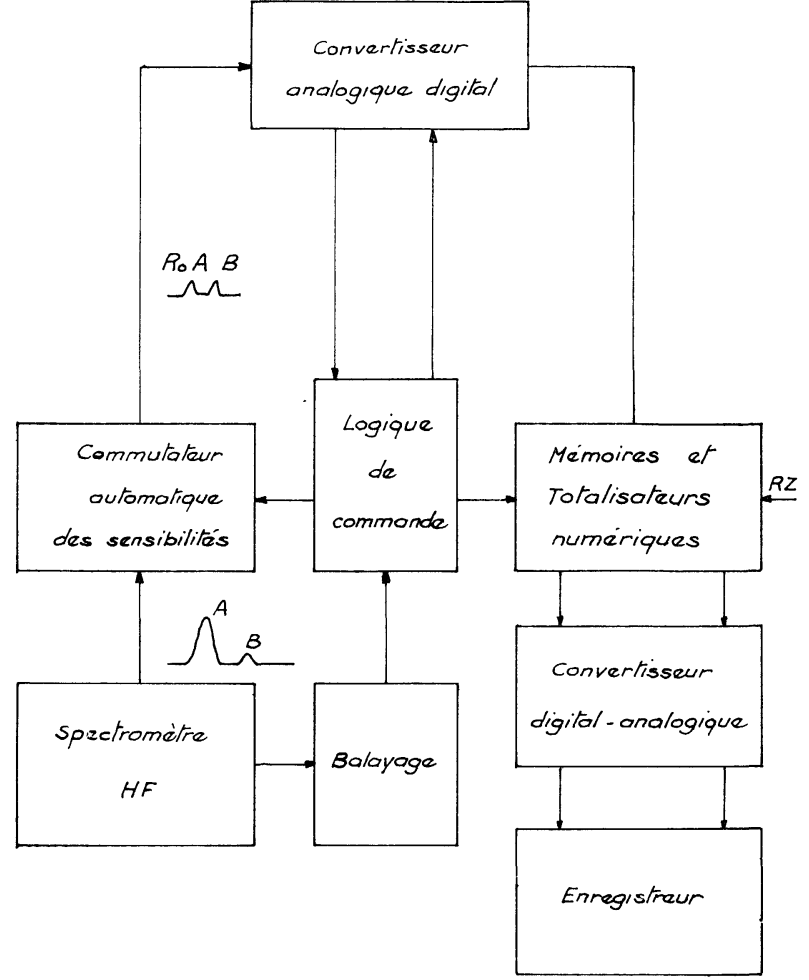

FIG. 6. - Schéma de principe du dispositif électronique de traitement d'information.



FIG, 7. - Schéma électronique de réalisation de la variante du dispositif de traitement d'information. 
transfert, de mise en mémoire des informations et de remise à zéro de l'appareil. L'ensemble de la logique est commandé par les pics eux-mêmes. Sans pærdre la généralité de l'emploi de l'appareil, nous allons décrire dans ce qui va suivre le dispositif électronique réalisé pour traiter deux pics isotopiques (fig. 7).

On décrira dans le premier paragraphe les sousensembles électroniques. La logique de commande qui règle les séquences de fonctionnement de l'appareil est exposée dans le second paragraphe.

1. Sous-ensembles électroniques. - a) BALAYAGE GYGLIQUE MAGNÉTIQUE. - Le spectromètre de masse travaille en simple collection avec balayage cyclique magnétique autour de deux masses dont on veut mesurer l'abondance isotopique.

La figure 8 montre le schéma électronique du système de balayage cyclique magnétique associé à l'alimentation stabilisée en courant de l'électro-aimant.

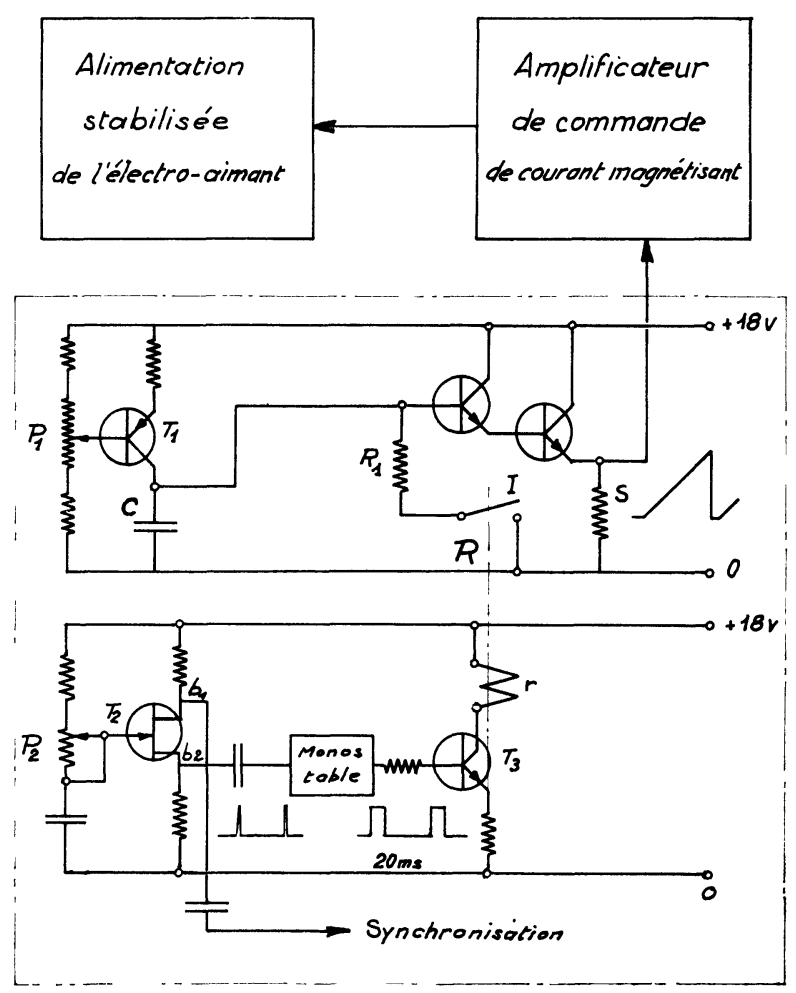

FIG. 8. - Alimentation stabilisée de l'électro-aimant et schéma électronique du balayage cyclique magnétique.

Ge système de balayage cyclique est constitué essentiellement d'un générateur d'oscillations de relaxation en dents de scie : c'est un circuit de charge et de décharge d'un condensateur. La charge du condensateur $\mathrm{C}$ est assurée par le transistor de puissance $\mathrm{T}_{1}$ dont le courant de commande de base est réglable par le potentiomètre $\mathrm{P}_{1}$. La décharge de ce condensateur est commandée par la mise en court-circuit du relais $\mathrm{R}$. La période de charge et de décharge du

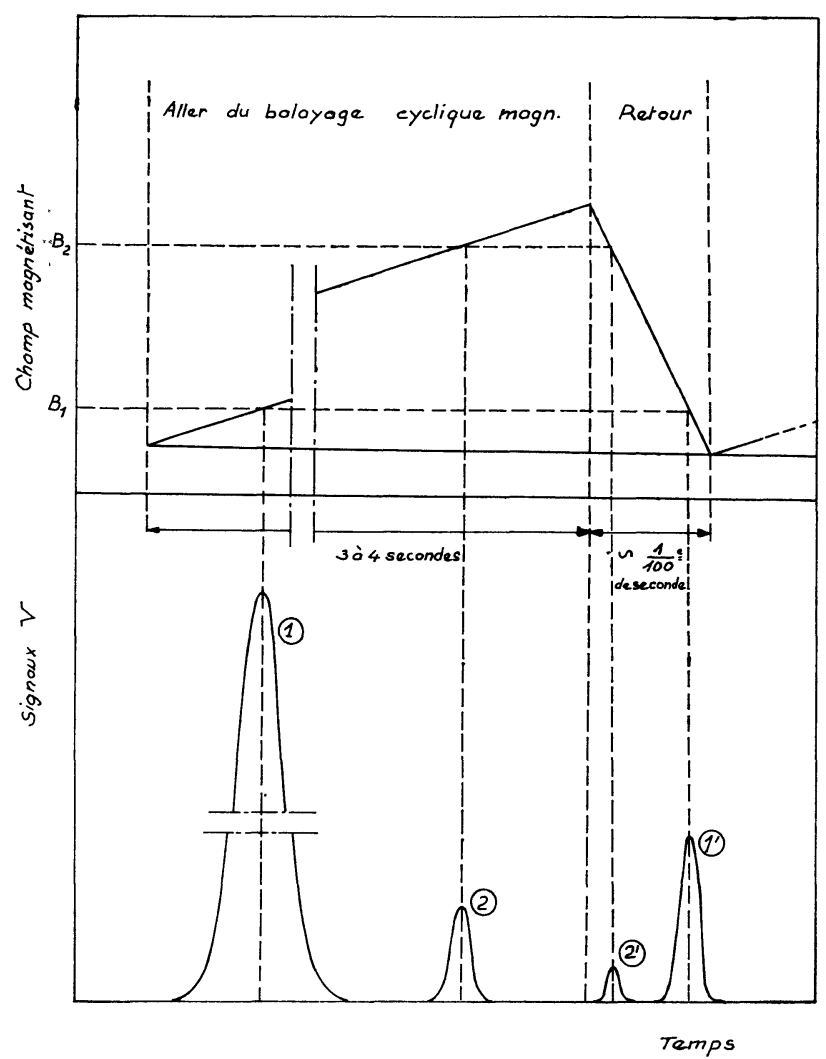

FIG. 9. - Représentation schématique des signaux délivrés par le spectromètre de masse.

condensateur, c'est-à-dire du balayage cyclique, est réglée par le transistor unijonction $\mathrm{T}_{2}$ monté en multivibrateur de période variable entre 0,5 et $5 \mathrm{~s}$.

La figure 9 schématise un cycle de balayage magnétique et les signaux correspondants que délivre le spectromètre de masse. Pour nos premières expériences de mise au point, nous prenons en général un cycle de l'ordre de $4 \mathrm{~s}$.

b) Commutateur de sensibilités. - Afin d'enregistrer les variations de $V_{\mathrm{A}}$ et $V_{\mathrm{B}}$, on atténue par un commutateur de sensibilités $R_{0}$ fois $V_{\mathrm{A}}$, de telle sorte que :

$$
V_{\mathrm{A}}=\frac{V_{\mathrm{B}}}{R_{0}}=V_{\mathrm{B}}^{\prime}
$$

$R_{0}$ étant le rapport isotopique de l'échantillon initial.

Le commutateur automatique de sensibilités ( $f i g .7$ ) comporte essentiellement un potentiomètre 10 tours de précision $P$ et un relais RGS. Le potentiomètre $P$ constitue le pont diviseur de tension et permet de rendre les deux pics rigoureusement égaux avant la conversion analogique-digitale. Le relais RCS dont la bobine de commande est excitée ou désexcitée séquentiellement se met dans la position de travail ou de repos. Il permet ainsi de prendre soit une partie du grand signal, soit la totalité du petit signal alternativement. 
c) Convertisseur analogique-digital. - G'est un convertisseur analogique-digital classique « par réaction ", dans lequel un compteur binaire compte des impulsions émises par un générateur de fréquence constante pendant l'intervalle de temps qui sépare l'instant origine et l'instant où une tension variable $V_{\mathrm{r}}$ progressivement croissante vient égaler la tension à coder $V_{\mathrm{e}}$ (fig. $10 \mathrm{~b}$ ). Le nombre d'impulsions obtenu fournira une mesure de la tension à convertir. La figure 10 a représente le schéma synoptique de ce
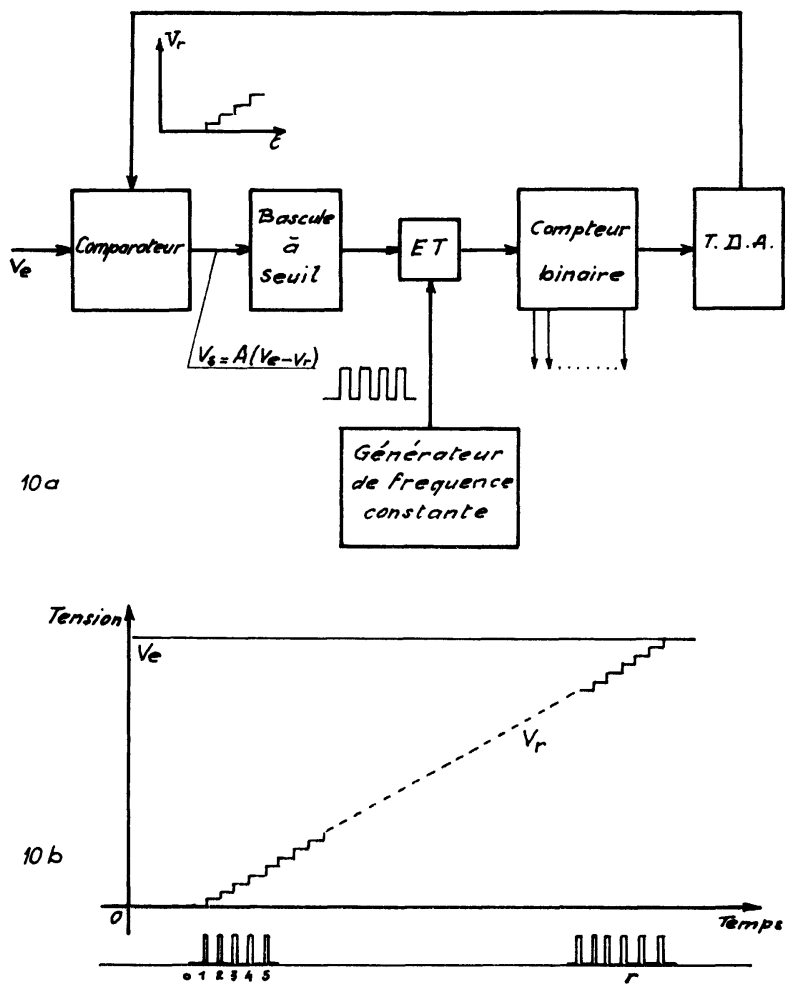

FIG. 10.

10 a Schéma de principe du convertisseur analogiquedigital ;

$10 \mathrm{~b}$ Représentation des informations à coder $\mathrm{V}_{\mathrm{e}}$ et de référence $V_{r}$.

convertisseur. Il comprend : un comparateur, une bascule à seuil ( «trigger »), une porte ET, un générateur de fréquence constante, un compteur binaire et un traducteur digital-analogique. Les caractéristiques de ce convertisseur sont : temps d'opération maximal : $40 \mathrm{~ms}$, précision maximale de conversion : $1 \%$, signal d'entrée maximal : $25 \mathrm{mV}$.

La précision de conversion maximale de $1 \%$ impose un compteur à 10 bascules binaires $\mathrm{FF}_{1}$ montées en cascade définissant $2^{\mathbf{1 0}}$ combinaisons et constituant une échelle allant de 0 à 1023 ( fig. 11). Pour coder des signaux d'entrée de $25 \mathrm{mV}$, il faut avoir des échelons unitaires de tension de 25/1 023, soit sensiblement égaux à $25 \mu \mathrm{V}$.



FIG. 11. - Schéma du compteur binaire et $d u$ convertisseur digital-analogique.

Le traducteur analogique-digital associé au compteur binaire comporte dix transistors travaillant en générateur de courant et un réseau en échelle de résistances de précision. Les courants et les résistances sont choisis de telle sorte que la tension $V_{\mathrm{r}}$ augmente par paliers de 25 en $25 \mu \mathrm{V}$ (fig. 11).

Le « trigger » a un seuil de basculement de $0,5 \mathrm{~V}$, valeur qui détermine le gain $A$ du comparateur égal à 20000 . Le comparateur est un amplificateur différentiel symétrique entièrement transistorisé à deux entrées et deux sorties. Il a un gain en boucle fermée de 25000 , un seuil de $20 \mu \mathrm{V}$, une impédance d'entrée de $100 \mathrm{k} \Omega$ et une bande passante de $30 \mathrm{kHz}$.

Le générateur d'impulsions est un multivibrateur de fréquence de $25 \mathrm{kHz}$.

d) MÉmoires, totalisateurs et sorties analoGIQUES. - Les expressions digitalisées de l'information analogique des pics spectrométriques, fournies par le convertisseur analogique-digital précédent, sont prises en charge et conservées soit dans deux mémoires, soit dans deux totalisateurs. Les mémoires permettent le renouvellement des informations instantanées correspondant aux pics spectrométriques; les totalisateurs l'intégration des signaux dans le temps.

Chacune des mémoires est constituée par dix bascules binaires précédées d'une porte ET à deux entrées. L'une des entrées est reliée directement à la sortie 
des bascules du compteur, l'autre à la ligne « transfert ». Les expressions digitalisées des pics isotopiques contenues dans le compteur sont ainsi transférées aux mémoires correspondantes de façon parallèle. Elles sont ensuite converties en informations analogiques dans deux traducteurs digitaux-analogiques pour alimenter éventuellement des enregistreurs classiques. Une ligne de remise à zéro mémoire $(\mathrm{ZM})$ permet d'effacer le précédent contenu. La figure 12 représente une mémoire de dix bascules et son convertisseur digital-analogique associé.



FIG. 12. - Schéma de la mémoire et de sa sortie analogique.

Les totalisateurs sont en fait deux compteurs binaires composés de bascules montées en cascade, qui reçoivent en même temps que le compteur du convertisseur analogique-digital les impulsions provenant du multivibrateur de fréquence $25 \mathrm{kHz}$. Ils sont précédés chacun d'une porte ET ouverte ou fermée suivant que c'est l'un ou l'autre pic isotopique. Le totalisateur garde en mémoire le nombre d'impulsions reçues pour l'additionner au suivant. Ces totalisateurs sont suivis de deux traducteurs digitaux-analogiques classiques. L'ensemble comporte enfin une ligne de remise à zéro pour effacer le contenu une fois la mesure terminée.

2. Circuits logiques de commandes. - La logique de commande est constituée par des éléments classiques en technique binaire ( fig. 7). C'est un ensemble qui distribue séquentiellement tous les ordres de : codage, commutation de sensibilités, correction des erreurs de commutation, remise à zéro des mémoires $(\mathrm{ZM})$, transfert aux mémoires (TM), remise à zéro du compteur (ZG) et blocage du codage pendant le retour de balayage.

Ces différents ordres sont donnés par les pics à l'entrée, ce qui rend le dispositif électronique de traitement d'information complètement indépendant du spectromètre de masse. Ils sont déclenchés uniquement par des combinaisons de 4 variables de commande, dont deux primaires $t_{1}$ et $t_{2}$ et deux secondaires $t_{3}$ et $t_{4}$. Le diagramme de temps de ces variables est représenté sur la figure 13 où les signaux lents sont de l'ordre de la seconde, les impulsions de l'ordre de la microseconde et les délais de l'ordre de $10 \mathrm{~ms}$.

Le comparateur dont on dispose possède deux sorties. L'une des sorties sensibilise le trigger T1 dès

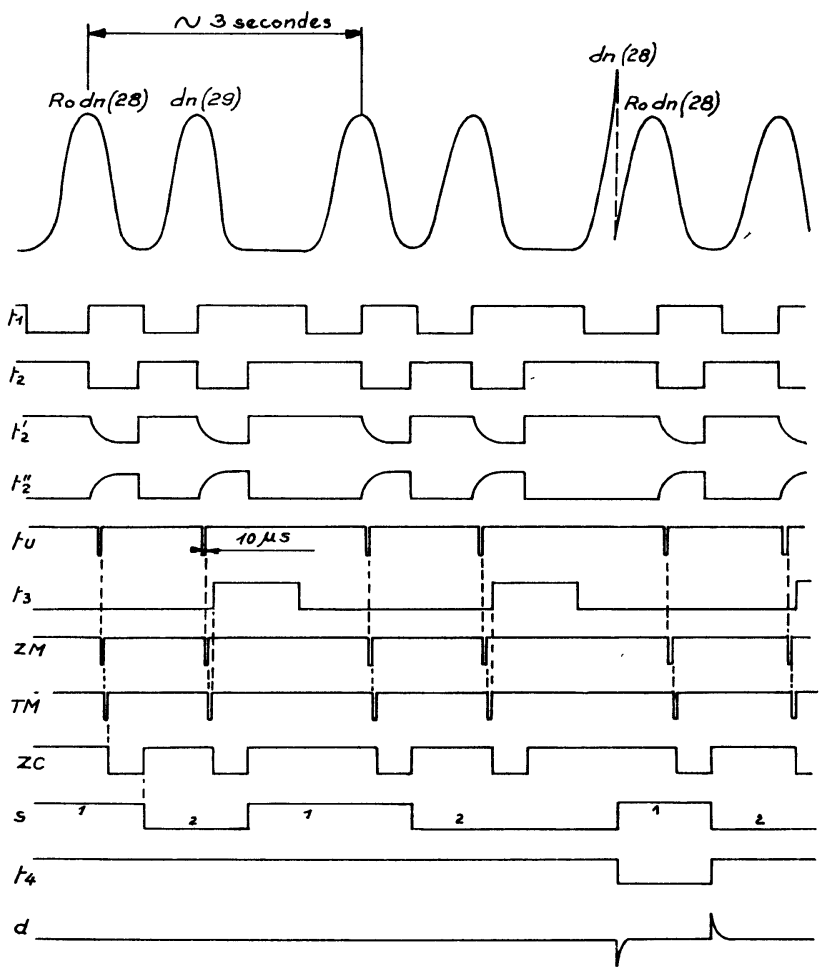

FIG. 13. - Diagramme de temps de la logique de commande de la variante du dispositif électronique de traitement d'information.

que le signal à l'entrée diffère de la tension de référence $V_{\mathrm{r}}$ de $25 \mu \mathrm{V}$ pour autoriser éventuellement l'opération de codage. L'autre sortie sensibilise le trigger T2 dès que le signal à coder est inférieur à $V_{\mathrm{r}}$ d'une quantité de $25 \mu \mathrm{V}$. Ainsi, la variable $t_{1}$ permet la conversion analogique-digitale, la variable $t_{2}$ commande toutes les opérations de traitement d'information digitalisée. La variable $t_{3}$ dérivée de $t_{2}$ est donnée par la monostable MS1 de retour de balayage cyclique. La variable de contrôle $t_{4}$ est fournie par la onzième bascule $\mathrm{BC}$ en série avec la dixième du compteur.

Quand l'opération de codage est terminée, la variable $t_{2}$ apparaît à la sortie du trigger T2 ( fig. 7$), t_{2}$ est 
intégré dans un circuit RG dont la constante de temps est réglable entre 10 et $100 \mathrm{~ms}$, ce qui permet d'éliminer les actions perturbatrices des parasites d'amplitudes suffisamment grandes mais de durée très brève. Le signal intégré $t_{2}^{\prime}$ de $t_{2}$, inversé par l'amplificateur inverseur AI, déclenche la monostable MS2 pour fournir l'impulsion de commande utile $t_{\mathrm{u}}$. La présence de $t_{3}$ à la porte ET2 permet le passage de $t_{\mathrm{u}}$ pour les pics considérés et interdit le passage pendant le retour $\mathrm{du}$ balayage cyclique. Le signal $t_{\mathrm{u}}$ déclenche trois monostables en cascade MS3, MS4, MS5 pour donner des ordres $Z M, T M$ et $Z G$ décalés les uns par rapport aux autres. Les impulsions ZM et TM s'appliquent respectivement aux portes ET3 et ET5, ET4 et ET6.

L'une des sorties Q1 de la bistable BS est reliée directement à ET3, ET4 et ET7, l'autre sortie Q2 à ET5, ET6 et ET8. De cette manière, on prédétermine et on sélectionne la mémoire $\mathrm{M} 1$ et le totalisateur TO1 pour le pic 1 et la mémoire M2 et le totalisateur TO2 pour le pic 2. En effet, si on choisit initialement l'état 0 de Q2 correspondant à la position $1 \mathrm{du}$ relais RGS (pour le pic 1), Q1 est à l'état 1 et les opérations de ZM, de TM et de totalisation peuvent s'effectuer pour la mémoire M1 et le totalisateur TO1, mais non pour M2 et TO2. Inversement, le changement d'état de Q2 amène le relais RCS à la position 2 dont le transfert de l'expression digitalisée du pic 2 ne peut s'effectuer que dans la mémoire M2 et les impulsions correspondant au pic 2 ne peuvent être comptées que dans le totalisateur TO2. De ce fait, on a un aiguillage absolument sûr des informations à mettre en mémoire.

L'impulsion ZC sortant de la troisième monostable MS5 mise en forme par MF2 sert à faire la remise à zéro du compteur et de la bascule $\mathrm{BC}$ et à changer les états de la bascule BS.

On voit que le relais du commutateur de sensibilités RCS est à la position 1 pour le grand pic isotopique durant toutes les opérations de conversion, de mise en mémoire et de totalisation. Il se met à la position 2 pour le petit pic 2 quand tout est fini et que le compteur est remis à zéro.

L'aller du balayage cyclique magnétique se faisant à vitesse lente permet au spectromètre de masse de fournir deux valeurs précises des signaux isotopiques 1 et 2 ; le retour s'effectuant à vitesse plus rapide, le spectromètre ne fournit qu'une fraction de pics $1^{\prime}$ et $2^{\prime}$ ( $f$ g. 9). Celles-ci sont considérées comme informations parasites et sont à éliminer. Pour ce faire, on emploie la variable $t_{3}$ qui ferme simultanément ET1 et ET2 durant ce retour. On verrouille ainsi le codage, la mise en mémoire, la totalisation et les différents ordres (voir diagramme de temps, fig. 13).

La bascule $\mathrm{BC}$ mise en cascade avec la dixième bascule du compteur constitue un système de sécurité qui contrôle le fonctionnement du commutateur de sensibilités. On a représenté sur la figure 13 le relais RCS toujours en fonction 2 après la fin des opérations utiles sur le pic 2. Cela veut dire que le grand pic passera en entier sans être atténué. De sorte que, lorsque le compteur à dix bascules est d'abord complc̀tement rempli, une impulsion déclenche la onzièmc bascule. Le changement d'état de BC se répercute par $t_{4}$ sur la bascule BS qui, elle aussi, change d'état pour ramener instantanément le relais RCS à la position correcte 1 . Le pic 1 atténué est codé normalement et tous les ordres sont alors distribués correctement.

\section{GHAPITRE IV}

\section{APPLICATION AU DOSAGE EN CONTINU D'ISOTOPES PARTIELLEMENT SÉPARES PAR CHROMATOGRAPHIE GAZEUSE}

On s'est proposé de mesurer de manière continue et directe la répartition et le facteur d'enrichissement de deux isotopes partiellement séparés par chromatographie en phase gazeuse, le long du pic effluent, avec l'ensemble de notre appareillage.

L'information donnée par le spectromètre de masse à collecteur unique équipé d'un balayage cyclique magnétique d'un pic effluent de chromatographie est schématisée sur la figure 14 . Le collecteur voit défiler les masses A et B et l'on enregistre au cours du premier

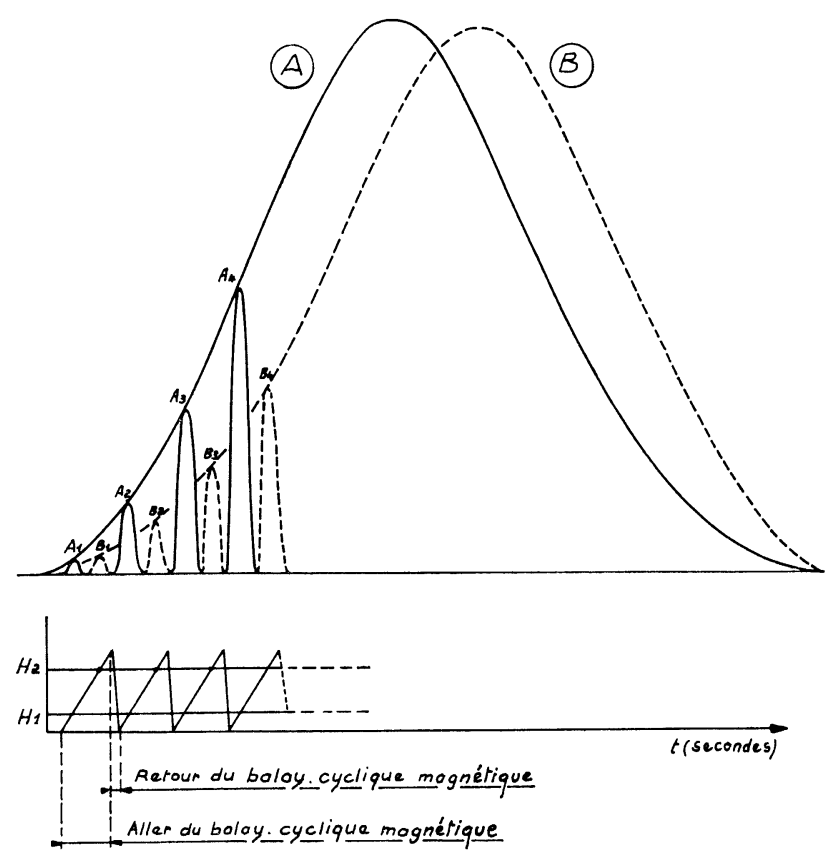

FIG. 14. - Représentation schématique des pics chromatographiques donnés par le spectromètre de masse à collecteur unique équipé de balayage cyclique magnétique.

cycle, par exemple, les signaux normalisés $A_{1}$ et $B_{1}$. La succession des signaux $A_{1}, A_{2}, \ldots, A_{\mathrm{p}}$ reproduit la variation d'amplitude du pic chromatographique 

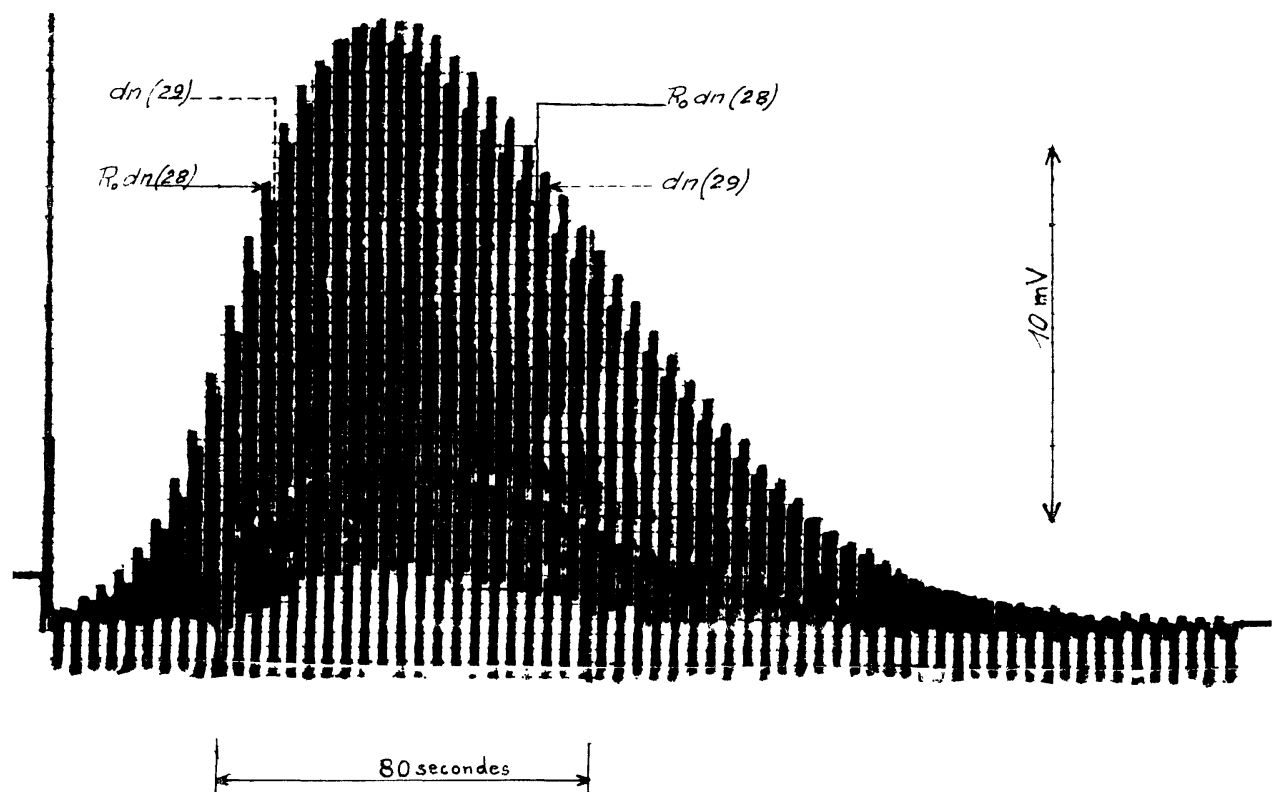

FIG. 15. - Reproduction photographique des signaux enregistrés à la sortie du commutateur automatique de sensibilités.

du constituant A. La somme des amplitudes $A_{1}+$ $A_{2}+\ldots+A_{\mathrm{p}}$ est proportionnelle à la surface de ce pic, c'est-à-dire au nombre de molécules $\mathrm{A}$ qui ont traversé le détecteur. Il en est de même pour le constituant B.

La mesure continue de la répartition des deux isotopes s'obtient par la différence instantanée des amplitudes $A$ et $B$ et le facteur d'enrichissement $\rho$ que nous avons défini dans un précédent article [12], par les intégrales des pics chromatographiques et leur différence [12].

Nous avons étudié la répartition des isotopes ${ }^{12} \mathrm{G}$ et ${ }^{13} \mathrm{C}$ du carbone obtenue par chromatographie en phase gazeuse de l'oxyde de carbone. Les conditions chromatographiques sont les suivantes : colonne de largeur $2 \mathrm{~m}$, de diamètre intérieur $0,4 \mathrm{~cm}$, et remplie de tamis moléculaire $5 \AA$ dont les grains sont entre $0,035 \mathrm{~cm}$ et $0,042 \mathrm{~cm}$; température $0^{\circ} \mathrm{C}$; volume d'échantillon $4 \mathrm{~cm}^{3}$ TPN; débit de gaz vecteur (hélium) $1 \mathrm{~cm}^{3} / \mathrm{s}$.

La figure 15 est une reproduction photographique des signaux enregistrés à la sortie du commutateur automatique de sensibilités réglé pour rendre égaux les pics isotopiques d'un échantillon standard naturel. Cette reproduction photographique doit être comparée avec la figure 14 : on y distingue une succession de traits verticaux juxtaposés donnant des signaux $R_{0} \mathrm{~d} n(28)$ et $\mathrm{d} n(29)$ toutes les trois secondes, $R_{0}$ étant le rapport isotopique naturel, $\mathrm{d} n(28)$ et $\mathrm{d} n(29)$ les nombres instantanés de molécules ${ }^{12} \mathrm{G}^{16} \mathrm{O}$ et ${ }^{13} \mathrm{G}^{16} \mathrm{O}$. On constate qu'au début du pic chromatographique les traits verticaux représentant des signaux $R_{0} \mathrm{~d} n(28)$ dépassent les traits verticaux représentant les signaux
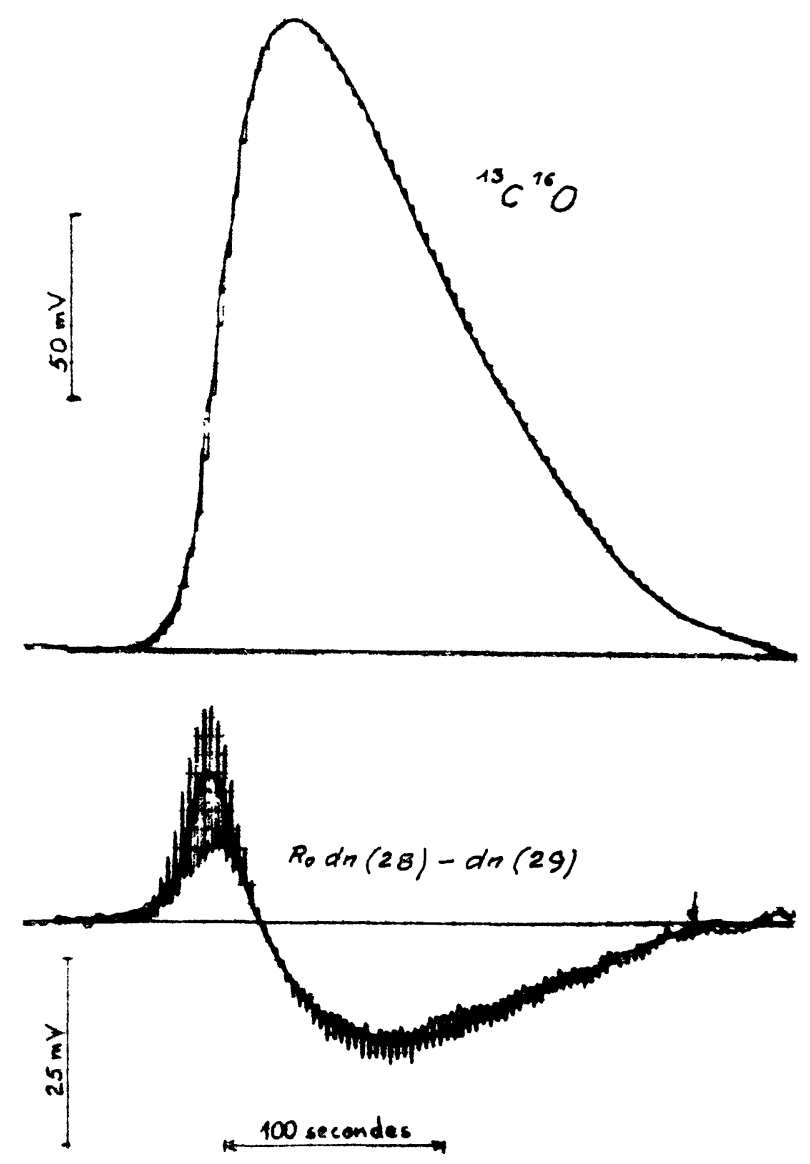

FIG. 16. - Reproduction photographique des signaux sortant du dispositif de traitement d'information. 
$\mathrm{d} n$ (29) qui, à leur tour, dépassent les traits $R_{0} \mathrm{~d} n(28)$ à la fin. Il est à noter que le pic chromatographique s'étale sur plus de $200 \mathrm{~s}$, ce qui permet de faire environ une soixantaine de cycles successifs de mesure.

La figure 16 est une reproduction photographique des signaux obtenus à la sortie des mémoires (dans le cas de l'enrichissement isotopique du carbone ${ }^{12} \mathrm{G}^{16} \mathrm{O}$ et $\left.{ }^{13} \mathrm{G}^{16} \mathrm{O}\right)$. On retrouve l'allure générale du pic chromatographique à la partie supérieure. L'enregistrement du pic d $n(29)$ présente une série de paliers caractéristiques de la technique digitale. En effet, l'information est mise en mémoire au cours de chaque cycle, dès que le codage est terminé. Le passage d'un cycle au suivant donne une nouvelle information, généralement différente de la précédente, ce qui conduit à un nouveau palier. Le signal $R_{0} \mathrm{~d} n(28)$ à la sortie de l'autre mémoire se présenterait de la même façon, les paliers étant sensiblement décalés dans le temps d'une demi-période. La partie inférieure de la figure 16 représente l'enregistrement de la différence des signaux $R_{0} \mathrm{~d} n(28)-\mathrm{d} n(29)$, enregistrement comparable à celui obtenu par la technique de double collection [13].

Pour calculer le facteur d'enrichissement $\rho$, la courbe moyenne tracée sur la figure 16 (partie inférieure) est utilisée. Chaque point de cette courbe est la valeur moyenne entre deux paliers, qui correspondent à un cycle de mesure. En effet, la mémoire M2, correspondant au pic chromatographique 29 , est décalée d'une demi-période par rapport à la mémoire $\mathrm{M} 1$, correspondant au pic 28, si bien que la différence $R_{0} \mathrm{~d} n(28)-\mathrm{d} n(29)$ présente deux paliers par cycle. L'écart entre ces deux paliers est d'autant plus marqué que les signaux varient au cours du cycle de mesure, et l'approximation médiane n'est exacte que dans la mesure où l'on a le même écart de temps entre les signaux 28 et 29 au cours d'un cycle et les signaux 28 et 29 entre deux cycles.

On trouve pour le facteur d'enrichissement :

$$
\rho=0,05 \pm 0,001
$$

en très bon accord avec les résultats trouvés dans la méthode de double collection [13].

On a aussi mesuré directement le facteur d'enrichissement isotopique du néon obtenu par chromatographie en phase gazeuse avec cet appareillage. Les conditions chromatographiques sont les suivantes : colonne de longueur $3 \mathrm{~m}$, de diamètre intérieur $0,4 \mathrm{~cm}$ et remplie de tamis moléculaire $5 \AA$ dont les grains sont entre $0,035 \mathrm{~cm}$ et $0,042 \mathrm{~cm}$; température 77 oK; volume d'échantillon $6 \mathrm{~cm}^{3} \mathrm{TPN}$; débit de gaz vecteur (hélium) $0,5 \mathrm{~cm}^{3} / \mathrm{s}$.

La figure 17 est la reproduction des signaux obtenus à la sortie des totalisateurs du dispositif électronique réalisé. La courbe supérieure donne à chaque instant le nombre d'atomes de néon 22 qui ont traversé le détecteur. Cette courbe a la forme caractéristique de l'intégrale d'un pic chromatographique. La seconde

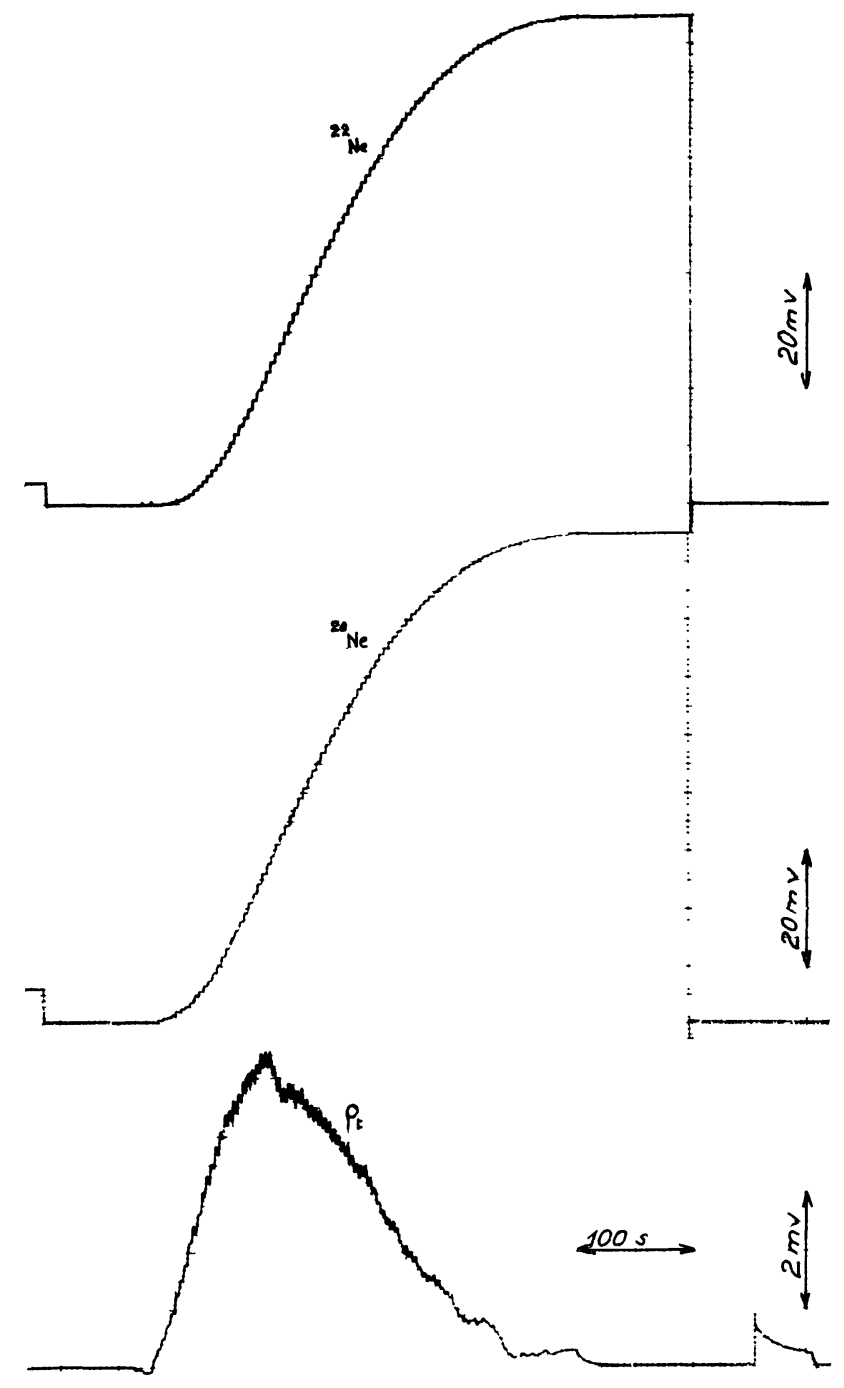

FIG. 17. - Reproduction des enregistrements des intégrales des pics correspondant aux isotopes ${ }^{20} \mathrm{Ne}$ et ${ }^{22} \mathrm{Ne}$ et $d u$ facteur d'enrichissement $p$.

courbe donne la même information néon 20. Cette courbe a la même forme que la précédente et l'atténuateur de sensibilités a été réglé en sorte qu'elle ait la même amplitude maximale. Chaque palier que l'on observe sur ces deux courbes représente un cycle de balayage magnétique et les deux paliers des deux courbes sont décalés d'une demi-période.

La courbe inférieure représente la différence des deux précédentes, c'est-à-dire la variation du facteur d'enrichissement [9].

On a trouvé :

$$
\rho=0,10 \pm 0,001
$$

en bon accord avec le résultat obtenu par la méthode de double collection [13]. 


\section{CONCLUSIONS}

Les résultats obtenus appellent deux remarques suivantes : l'une concernant le spectromètre de masse original, l'autre le dispositif électronique de traitement d'information.

Le spectromètre de masse que l'on a construit fournit grâce à la source d'ions à excitation électrique de haute fréquence des courants intenses qui permettent d'éviter l'emploi d'impédance de charge importante sur le collecteur, donc d'éliminer les dérives de l'appareillage et d'abaisser les constantes de temps. Les mesures qui en résultent s'avèrent d'autant plus précises et plus reproductibles.

Le dispositif électronique digital associé à ce nouveau spectromètre de masse, équipé d'un balayage cyclique magnétique et d'un collecteur unique, permet d'une part de travailler de manière beaucoup plus générale avec des corps à deux, trois, quatre, etc., isotopes, d'autre part d'avoir une seule chaîne de mesures pour différentes informations. Il permet d'enregistrer en continu la répartition des isotopes le long d'un pic effluent de chromatographie en phase gazeuse et de mesurer directement le facteur d'enrichissement isotopique.

Les résultats obtenus avec ce dispositif simplifié sont, en précision et en sensibilité, comparables à ceux du montage à double collecteur [13].

Enfin, l'ensemble de cet appareillage est simple, de fonctionnement sûr et commode; il peut être employé comme unité de mesure et de contrôle de la séparation isotopique par chromatographie en phase gazeuse.

Remerciements. - Nous remercions vivement M. N. Inchauspé, Directeur des Laboratoires de Chimie-Physique de la S.N.P.A., pour les conseils, les encouragements et l'aide qu'il nous a apportés dans la réalisation de ce travail.

\section{BIBLIOGRAPHIE}

[1] Blanc (D.) et Degeily (A.), J. Physique Rad., 1961, 22, $230 ;$ C. R. Acad. Sc. Paris, 1959, 248, 1647 J. Physique Rad., 1959, 20, 55 A ; C. R. Acad. Sc. Paris, 1950, 250, 3313 ; Ind. Atom. Genève, 1961 11, 12, 71 ; C. R. Acad. Sc. Paris, 1961, 253, 1682.

[2] BARnetT (C. F.) et RAY (J. A.), Atomic Collision Processes, Inst. Conf. on the Phys. of Elect. and Atomic Collisions, éd. John Wiley and Sons, Inc. London, 1963.

[3] Chambers (E. S.), Rev. Sc. Inst., 1965, 36, 41.

[4] AbBe (J. C.), Thèse de Doctorat de Spécialité, Strasbourg, 1962.

[5] AbBé (J. C.) et Adloff (J.), J. Physique Rad., 1963, 24, 6, 94 A.

[6] Garcia (J.), Thèse de Doctorat de Spécialité, Toulouse, 1966.
[7] Espagno (L.), Blanc (C.) et Enjalbert (A.), Brevet d'Invention français, no 982776 (déposé le 23 juillet 1964).

[8] Degeil.H (A.), Thèse de Doctorat ès Sciences Physiques, Toulouse, 1962.

[9] Huynh Chanh Trung, Thèse de Doctorat ès Sciences Physiques, Toulouse, 1966.

[10] BARNARD (G. P.), "Modern Mass Spectrometry ", The Inst. of Phys., London, 1952, p. 45.

[11] Bordenave-Montesquieu, Thèse de Doctorat de Spécialité, Toulouse, 1966.

[12] BLanc (C.), Huynh (C. T.) et Espagno (L.), J. Chrom., 1967, 28, 177-208.

[13] BLANC (C.), Thèse de Doctorat ès Sciences Physiques, Toulouse, 1966. 